\title{
Inklusionsarchitekturen: Wie wird ein Lernort zu einem inklusiven Lernort im öffentlichen Raum des Lebenslangen Lernens?
}

\author{
Silke Schreiber-Barsch • Emma Fawcett
}

Eingegangen: 29. Juli 2017 / Angenommen: 30. Oktober 2017 / Online publiziert: 17. November 2017 (C) Der/die Autor(en) 2017. Dieser Artikel ist eine Open-Access-Publikation.

Zusammenfassung Der Beitrag stellt die Frage in den Fokus, wie Inklusion im Sinne der UN-Behindertenrechtskonvention an Lernorten von Erwachsenen umgesetzt wird und präsentiert Ergebnisse einer explorativ-qualitativen Studie mit professionell Tätigen. Auf der Grundlage relationaler Raumtheorie ist ein Modell von Inklusionsarchitekturen von institutionalisierten Lernorten im öffentlichen Raum entwickelt worden. Gezeigt werden kann die Herstellung sozialer Räume im und durch das Handeln von professionell Tätigen und Konsequenzen für den Zugang zu Lernorten und dortigen Partizipations(an)ordnungen, insbesondere entlang der Kategorie „dis/ability“. Lernorte, so unser Resümee, eröffnen ein Brennglas auf einen alltäglichen Schauplatz der (konfliktären) Aushandlung von Zugehörigkeitsordnungen zur community der Lernenden.

Schlüsselwörter Inklusion · Raum · Löw · Öffentliche Erwachsenenbildung · Professionelle $\cdot$ Partizipation

\section{The architecture of inclusion: What renders a learning place into an inclusive learning place in the public space of lifelong learning?}

\begin{abstract}
The paper raises the question of how inclusion, in the sense of the UN Convention on the Rights of Persons with Disabilities, is operationalized in institutional learning settings for adults. It presents findings of a qualitative pilot study.
\end{abstract}

\footnotetext{
Jun.-Prof. Dr. S. Schreiber-Barsch ( $₫)$

Universität Hamburg, Hamburg, Deutschland

E-Mail: silke.schreiber-barsch@uni-hamburg.de

E. Fawcett, M.A., M.A. (Oxon.) ( $₫)$

Humboldt-Universität zu Berlin, Berlin, Deutschland

E-Mail: emma.fawcett@hu-berlin.de
} 
Based on a relational theory of space, the findings elicit a conceptual model of the architecture of inclusion at public places of learning for adults. The model illuminates the way social space is constituted in and by the actions of professionals, and the resulting consequences for access to public learning places and order/ings of participation there, in particular regarding "dis/ability". Thus, places of learning act as a magnifying glass on the daily arena of (contested) negotiations on belonging to the community of learners.

Keywords Inclusion $\cdot$ Space $\cdot$ Löw $\cdot$ Public adult education · Professionals · Participation

\section{Einleitung - zum Zusammenhang von Erwachsenenbildung, bildungspolitischer Inklusions-Agenda und relationaler Raumtheorie}

Mit der Umsetzung von Inklusion im Sinne der UN-Behindertenrechtskonvention (UN-BRK) (United Nations 2006) deute sich, so die Deutsche Gesellschaft für Erziehungswissenschaft, eine ,dritte Revolution“ (DGfE 2015, S. 1) im bundesdeutschen Bildungswesen und innerhalb der Erziehungswissenschaft an. Während der schulische Umsetzungskontext breit diskutiert wird, zeigt sich in der Erwachsenenbildung ein anders gelagertes Spannungsfeld aus Debatten um disziplinäre Nicht-/ Zuständigkeit, professionelles Nicht-/Handeln und individueller Nicht-/Teilnahme. Hier setzt der Beitrag an und fragt, wie Inklusion im Sinne der UN-BRK an Lernorten von Erwachsenen umgesetzt wird; präsentiert werden Ergebnisse einer explorativen Studie. Die Erhebung zielte darauf, die Räumlichkeit professionellen Handelns an als inklusiv deklarierten Lernorten Erwachsener nachzuvollziehen und zu verstehen, wie in und über dieses Handeln der öffentliche Raum des Lebenslangen Lernens für lerninteressierte Subjekte unter der Frage von dis/ability gestaltet und (neu) ausgehandelt wird. Damit wollen wir zur Ausdifferenzierung einer erwachsenenpädagogischen Position in den „Begründungs-“ und „Realisierungsdiskursen“ (Lindmeier und Lindmeier 2015) um Inklusion beitragen.

Einleitend ist anzuführen, dass der Begriff „Inklusion“ pluridisziplinär verankert und seit Jahrzehnten in sozialwissenschaftlichen Diskursen beheimatet ist, zumeist in dialektischer Verschränkung mit „Exklusion“ (vgl. z. B. Schreiber-Barsch 2015b; Schäffter und Ebner von Eschenbach 2015). Grundsätzlich im Verständnis zu unterscheiden ist ein gesellschaftliches System von Inklusion/Exklusion als eine sozialpolitische Sicherungs- und Distributionsstrategie, das sich materialisiert in kontinuierlich und parallel ablaufenden dialektischen Prozessen der sozialen Einund Ausschließung. Gesamtgesellschaftliche Veränderungsbedarfe bzw. Reproduktionswünsche begründen sich entlang normativer Leitvorstellungen, die sich an der Vision eines gesellschaftlichen Zustandes orientieren, wie bspw. dem einer inklusiven Gesellschaft (BMAS 2011, 2016; Schreiber-Barsch 2015b). Letzteres illustriert die gegenwärtige Überlagerung: Während der Anspruch, soziale Inklusion von Erwachsenen qua Bildung und Lernangeboten $\mathrm{zu}$ befördern und strukturell zu gewährleisten, zu den historischen Leitmotiven der Erwachsenenbildung gehört, fokussieren Inklusion im Sinne der UN-BRK und die nationale Inklusions-Agenda 
(BMAS 2011, 2016) diesen Anspruch auf die Kategorie dis/ability (s. Kap. 2.1) und fordern darunter die Umsetzung des gültigen Rechts auf Zugang zu Bildung und auf gemeinsames, selbstbestimmtes Lernen. Hier setzt der Beitrag an: Soziale Inklusion prozessiert sich im System des Lebenslangen Lernens qua Bildung und Lernen und materialisiert sich u. a. an inklusiven Lernorten, d. h. Lernorten, die auf der Grundlage normativer Leitvorstellungen einen diskriminierungsfreien Zugang und diversitätsbewusste Lehr-Lernkontexte bieten - und dies u. a. für erwachsene Lerninteressierte mit Behinderungen.

Die festzustellende Dynamik in Bildungspraxis und -diskurs der vergangenen Jahre unter dem Stichwort Inklusion bedingt sich vor allem aus dem deklarierten Veränderungsbedarf auf Systemebene. Seitdem wirkt Inklusion als bildungspolitische Interventionsstrategie (Schäffter und Ebner von Eschenbach 2015, S. 232) auf das gesamte Mehrebenensystem der Weiterbildung ein (Schrader 2011): Die Makroebene supranationaler Bildungspolitik (Ratifizierung der UN-BRK in Deutschland im Jahre 2009) findet ihre Implementierung auf der nationalen Ebene der bildungspolitischen Inklusions-Agenda (BMAS 2011, 2016). Auf der Mesoebene sind die institutionellen Akteure des Bildungssystems aufgefordert, Kindern, Jugendlichen und Erwachsenen einen diskriminierungsfreien Zugang zu einem ,,inclusive education system at all levels and lifelong learning" zu sichern (United Nations 2006, S. 16). Gleichwohl trennen sich hier die Logiken von Schule bzw. Erwachsenenbildung: Schule funktioniert im Modell der Bildungspflicht und operiert mit einer nahezu festgelegten Grundgesamtheit an Schülerinnen und Schülern; hingegen ist konstitutives Element der Erwachsenenbildung das Kernprinzip der Freiwilligkeit von Teilnahme an den Angeboten. Diese andere Logik wirkt auf der Mikroebene der institutionellen Binnenstrukturen und der erwachsenenpädagogischen Lehr-Lernsettings in das Angebot-Nachfrage-Gefüge fundamental hinein. Es bestehen strukturell Freiräume für Disziplin, Träger, professionell Tätige, für Lehrende und Lernende, ob, und wenn ja, inwiefern sie inklusive Lehr-Lernsettings anbieten, pädagogischprofessionell ausgestalten, wissenschaftlich bearbeiten oder auch als Lernende an ihnen teilnehmen.

Um die Ausdeutung und Gestaltung dieser Freiräume in der Erwachsenenbildung zu erfassen, haben wir die Perspektive von professionell Tätigen gewählt und in unserer Erhebung gefragt, wie Inklusion an institutionalisierten Lernorten Erwachsener von dort pädagogisch Tätigen operationalisiert wird, die nicht lehrend, sondern hauptsächlich planend-disponierend arbeiten. Kurzum: Was macht aus Sicht von Professionellen den Lernort zu einem inklusiven Lernort? Pädagogische Institutionen sind im Bildungssystem als eine ,inklusionsvermittelnde Instanz“ (Hertel 2013, S. 157) vorgesehen an der Scharnierstelle zwischen Subjekten und dem Teilhabeund Statusversprechen durch Bildung. Die bildungspolitische Interventionsstrategie setzt diesbezüglich die normative Leitvorstellung, inwiefern eine Wirkung auf institutionelle Rahmenbedingungen und auf ,pädagogische Handlungsorientierungen und -logiken“ (Nuissl von Rein und Dollhausen 2011, S. 118) erfolgen soll. In diesem Übersetzungsvorgang kommt hauptamtlich Tätigen und ihrem planenddisponierenden Handeln eine Schlüsselstellung für die Operationalisierung von Inklusion zu. „Operationalisierung“ verwenden wir in dem Sinne, dass wir nach einer 
Präzisierung der pädagogisch-professionellen Umsetzungsverfahren und -logiken an dem je konkreten, physisch-materiellen Lernort gefragt haben.

Hierbei diente uns als Ausgangsthese, dass die Transformation zu einem inklusiven System des Lebenslangen Lernens weder allein didaktisch (inklusive Methodik und Vermittlung) noch rein programmatisch (Leitbildmetaphern, öffentliche Bildungskampagnen), noch allein physisch-materiell (Enthinderung durch barrierearme Infrastrukturen mit bspw. dem Einbau eines Fahrstuhls) beantwortet werden kann. Diese Vielschichtigkeit bildet sich durchaus im (insgesamt überschaubaren) Wissenschaftsdiskurs zu Erwachsenenbildung und Inklusion ab (z. B. Kronauer 2010; DIE 2012; Burtscher et al. 2013; HBV 2015); eine zusammenführende Systematisierung und Weiterentwicklung steht aber noch aus, genauso wie eine Verschränkung mit Praxisdiskursen (s. z. B. Zeitschrift „Erwachsenenbildung und Behinderung“ - ZEuB) oder mit dem schulischen Diskurs. Darüber hinaus erfordert die Auseinandersetzung mit Inklusion, so unsere Überzeugung, die Anerkennung als ein mehrschichtiges pädagogisches Wirkungsgefüge aus Subjekten, Strukturen, Symbolik und Materialität, eingelagert in machtdurchsetzte gesellschaftliche Verhältnisse. Erkenntnisgenerierend erschienen uns deshalb raumanalytische Forschungsperspektiven als theoretische Rahmung und methodisches Analyseinstrumentarium, insbesondere relationale Raumtheorien in Form handlungstheoretischer Konzeptionen (Löw 2001) und der sozialwissenschaftliche Diskurs um soziale Räume (Kessl und Reutlinger 2010a). Zu dieser Verschränkung von Raum, Inklusion und dis/ ability finden sich einzelne Arbeiten im schulischen Kontext, bspw. zu der Rolle von Integrationshelferinnen und -helfern in Schulklassen (Köpfer 2014), oder aus soziologischer Perspektive als ethnografische Feldstudie über ein inklusiv betriebenes Hotel (Modes 2016); zu Erwachsenenbildung, Raum und Inklusion im Sinne von dis/ability hingegen nur erste Ansätze (Schreiber-Barsch 2015a; Haar 2014).

\section{Untersuchungsfokus: Lernorte im öffentlichen Raum und die Kategorie dis/ability}

Im erwachsenen-/bildungswissenschaftlichen Diskurs ist die Kategorie Raum jüngst erneut rezipiert und konzeptionell weiter ausdifferenziert worden (vgl. z. B. Bernhard et al. 2015; Franz 2016; Zeitschrift für Pädagogik 2016; Ebner von Eschenbach 2017; Der pädagogische Blick 2017). Der Anschluss an relationale Raumtheorien, insbesondere an die Arbeiten von Löw (2001), bietet ein Verständnis von Raum als einer Verschränkung von materiellen, soziostrukturellen und symbolischen Komponenten, die Raum als eine ,relationale (An)Ordnung sozialer Güter und Menschen (Lebewesen) an Orten“ (ebd., S. 224; Herv. i. Orig.) fassen. Dieses Verständnis überwindet bspw. rein territorial verstandene Operationalisierungsmuster von Inklusion im Sinne eines „Dabei-Sein[s] [an Lernorten] ist alles“ (Schreiber-Barsch 2016) und damit Verengungen formalistischer Raumvorstellungen (Kessl 2016) hin zur Relationalität von Räumlichkeit als topologischer Perspektive (Ebner von Eschenbach 2017). Mit dem Moment der Lokalisierung der bildungspolitischen InklusionsAgenda auf dem Territorium eines Lernorts eröffnen sich Optionen: für professionell Tätige ein pädagogischer Gestaltungszugriff auf die Option inklusiver Lehr- 
Lernsettings, für lerninteressierte Subjekte Spielräume für Teilnahme an Angeboten und einer Partizipation am Lernort und für Forschende raumanalytische Verstehenszugänge auf Prozesse der Herstellung und Veränderung sozialer Räume. Letzteres ist Ausgangspunkt des Beitrags.

\subsection{Hegemoniale Formationen des öffentlichen Raums des Lebenslangen Lernens}

Inklusion im Sinne der UN-BRK (UN-BRK 2006) und die daran gekoppelte Inklusions-Agenda zielen auf die Kernlogik des deutschen Bildungssystems. Diese hat über Jahrhunderte entlang der Differenzlinie fähig/nicht-fähig, dem ,able/not-able divide" (Campbell 2009), sowohl im schulischen als auch im erwachsenenpädagogischen Bereich sozial segregierend und territorial separierend gearbeitet (vgl. Schreiber-Barsch 2016, 2017; Schreiber-Barsch und Pfahl 2014). Aus raumanalytischer Sicht erkennen wir Raumordnungen: Über Raumordnungen werden gesellschaftliche Normalitätserwartungen und Konventionen hinsichtlich dessen, wie bestimmte Räume auszusehen hätten, wer diese nutzen dürfe, welche symbolische Wertigkeit ihrem territorialen Ort zugesprochen wird usw., in Regeln und Institutionen eingeschrieben und durch Ressourcen abgesichert (Kessl und Reutlinger 2010a, S. 249; Löw 2001, S. 245). Erkennbar wird eine ,hegemoniale Räumlichkeit“ (Kessl und Reutlinger 2010b, S. 251), also ,welche räumlichen Zusammenhänge zu einem bestimmten historischen Zeitpunkt gewollt und welche nicht gewollt sind“ (ebd.).

Solche hegemonialen Verhältnisse sind in den Raumordnungen im System des Lebenslangen Lernens in Bezug auf Menschen mit bzw. ohne Behinderungen abzulesen. Das Merkmal Behinderung bzw. dis/ability - als eine von vielen Kategorien sozialer Ungleichheit - verweist auf erwachsene Lerninteressierte mit kognitiven, körperlichen, sensorischen und/oder seelischen Behinderungen. „Behinderung“ verstehen wir im Anschluss an die Critical Disability Studies als ein kulturelles Modell (Shakespeare 2014; Snyder und Mitchell 2006; Riddell und Watson 2014), d. h. ein Wechselspiel aus sozialen Strukturen und Praktiken, individuellen Haltungen und körperlichen Wahrnehmungen sowie gesellschaftlich-kulturellen Normalitätserwartungen. Prozesse hegemonialer Räumlichkeit zeigen sich in der relationalen (An-)Ordnung (Löw 2001), in der seit Mitte des 18. Jahrhunderts die Raumordnung Sonder-Räume für erwachsene Lernende mit Behinderungen (Schreiber-Barsch 2015a) über diskursive pädagogische Praktiken normativ legitimiert wurde und sich in separierten bzw. segregierten Lernorten räumlich manifestiert(e) (Theunissen und Hoffmann 2003; Heimlich und Behr 2009; Powell und Pfahl 2012; Hinz 2013). Diese Raumordnungsstrukturen wirken bis heute. So ist bspw. quantitativ gesehen der „übliche“ Lernort von erwachsenen Lernenden mit Behinderungen weiterhin eine Einrichtung der Behindertenhilfe ohne primären Erwachsenenbildungsauftrag (Bsp. Werkstätten), nicht aber die örtliche Volkshochschule (Lindmeier 2003; Ackermann 2012). Diese räumliche Formation von Einschluss und Ausschluss als etwas ,objektiviertes Soziales“ (Frey 2004, S. 221) muss ihre Ausschließungen nicht zwangsläufig über Verbote durchsetzen. Ausschluss kann über Selbstausschluss hochwirksam „funktionieren“; so stellt im Sozialraum bspw. die Volkshochschule um die Ecke für die einen den üblichen Lernort und für andere (trotz gleicher physischer Nähe) 
eine ferne Welt dar (vgl. Löw 2001, S. 215; Bremer et al. 2015; Schroer und Wilde 2010, S. 187).

Diese traditionelle Raumordnung wurde infolge der Ratifizierung der UN-BRK im Jahr 2009 umdeklariert zu einer „Unordnung“ (Fritsche 2010), d. h. ausgewiesen als problematisch im Abgleich zum Leitmotiv einer ,inklusiven Gesellschaft“ (BMAS 2011) und als mit Maßnahmen zu bearbeiten. Der Steuerungsimpuls der Inklusions-Agenda lässt sich im Anschluss an Kraus (2015a) beschreiben als ein bildungspolitisches Instrument der Raumplanung pädagogischer Räume mit dem Ziel, Lernen zu ermöglichen. Raumplanung diene u. a. einer „Ausgleichsfunktion“ (ebd.), über die potenziell konfliktäre bzw. sozial ungleich gelagerte Zuweisungen, Interessen oder auch Ansprüche an die dann getätigte Nutzung eines pädagogischen Raums verhandelt werden (ebd.). Dies ist für unsere Fragestellung wesentlich, da sich hierin die öffentliche Verantwortung für eine sozial ausgleichende, zugleich politisch zu kontrollierende Gestaltung des öffentlichen Raums des Lehrens und Lernens widerspiegelt. Öffentlich wird ein Raum über das eingelagerte Prinzip der allgemeinen Zugangs- und Nutzungsmöglichkeit (Frey 2004, S. 223). Entscheidend ist jedoch: Es geht zwar auch um die territoriale Dimension eines prinzipiell für jede Person betretbaren Lernorts, seine Wirkmächtigkeit als öffentlicher Raum (vgl. Fritsche 2010, S. $57 \mathrm{f}$.) liegt jedoch in den sozial ungleich verteilten und individuell unterschiedlich erfahrenen Chancen und Möglichkeiten seiner Aneignung und Nutzung. Wirkmächtigkeit generiert sich über Prozesse der je individuell subjektiven, atmosphärischen Wahrnehmung der Zugänglichkeit des Ortes (vertraute Welten - fremde Welten), seiner symbolischen Aufladung (dabei-sein am Lernort ist statuserhöhend - statusherabsetzend) oder auch der strukturellen Beschaffenheit der Angebote für Nutzung und Aneignung (einschließend - ausschließend). Erst durch die je spezifisch getätigten Nutzungen von Aneignungsangeboten werden solche Wahrnehmungen und Erfahrungen individuell wie kollektiv aktiviert (Rau 2013, S. 184).

Das in Räumen und in ihren Konstituierungsprozessen inhärente Prinzip der Verteilung eröffnet Aufschluss über Macht- und Herrschaftsverhältnisse (Löw 2001, S. 214; Löw et al. 2008, S. 65; Kessl und Reutlinger 2010a). Erkennbar wird die sozial ungleich verteilte „Geltungsmacht“ (Löw 2001, S. 245) der Akteure, sich Räume aneignen, ihre Nutzungsstrukturen verhandeln bzw. sie eigentätig umeignen (Deinet 2010) zu können. Es werden potenziell konfliktäre Interessen, Ansprüche und Zuweisungen an die Nutzung eines pädagogischen Raums und ergo organisationale wie subjektive Handlungsfähigkeiten und -optionen verhandelt. Der Zugang zu sozialen Gütern wie einem Lernort reproduziere sich, so Löw (2001, S. 212 ff.), neben distributiven Ungleichheiten primär über ,relationale Formen sozialer Ungleichheit“" (in Anschluss an Kreckel 1992); Ungleichheiten minderten Zugang entlang von „hierarchischer Organisation (Rang)“ oder auch „selektiver Assoziation“ im Sinne der assoziativen Zugehörigkeitszuweisung zu einer Gruppe. Löw schlägt eine Prüfung dieser Assoziations-Dimension für die Analyse der Konstitution von Räumen in Bezug auf soziale Ungleichheit vor: Also die Prüfung der Minderung bzw. Begünstigung der Chancen auf Raumkonstitution auf der Grundlage von Zugehörigkeit bzw. Nicht-Zugehörigkeit zu sozialen Gruppen (Löw 2001, S. 214), was sich als anschlussfähig für unsere Fragestellung erweist. 


\subsection{Räumlichkeit professionellen Handelns an institutionalisierten Lernorten}

Während Kursleitenden in den Binnenstrukturen der Lehr-Lernsettings die Schlüsselbedeutung für die Frage von Vermittlung und einer Umsetzung von Inklusion zukommt sowie Teilnehmenden die Frage der Aneignung der Vermittlungsangebote, fokussieren wir den diesen Binnenstrukturen vorgängigen Handlungsraum der Planung und Organisation und schließen hier an den Diskurs um Lernorte an (vgl. zum Überblick Kraus 2015a). Lernorte bieten den territorialen Schauplatz der (potenziell konfliktären) Aushandlung von Nutzungsinteressen bzw. -ansprüchen an pädagogische Lernräume (Kraus 2015b). Bei uns geht es um intentionale Lernorte (ebd.), die eine institutionalisierte, öffentlich zugängliche Ermöglichungsstruktur anbieten und dem Ort einen bestimmten Grad von Vermittlungsleistung bzw. Lernförderlichkeit unterstellen, der im öffentlichen Raum und über professionelles Handeln eine „organisierte Einwirkungsabsicht auf den Bildungsgang von Erwachsenen“ sicherstellen soll (Kade et al. 2007, S. 132).

Aus raumanalytischer Perspektive stehen professionell Tätige in der Gestaltungsverantwortung für je konkrete Lernorte Erwachsener und beteiligen sich mit ihrem pädagogischen Planungshandeln wirkmächtig an der (Re-)Produktion von inklusiven Lernräumen. Es werden die Formationen hegemonialer Räumlichkeit (s. oben) dahingehend erkennbar, wer aus pädagogisch-professioneller Sicht derzeit als bildungsfähig und als bildungsbedürftig definiert wird und demzufolge als zu inkludierender Adressat Angebote der Erwachsenenbildung gilt - mit den daraus folgenden Mechanismen von Einschließung bzw. Ausschließung, von Zuweisungen zu je spezifischen Orten im System des Lebenslangen Lernens. Professionell Tätige nutzen, wie zu sehen sein wird, ein Spektrum an Handlungsmöglichkeiten in der Herstellung inklusiver Räume (auch ein Nicht-Handeln ist in diesem Sinne Handeln, ein unterlassendes Handeln). Hierbei ist ihr Handeln weder rein determiniert, noch rein willkürlich; es ist eingelagert in „orientierende Wissensbestände“ (Nuissl von Rein und Dollhausen 2011, S. 121). Es orientiert sich an gängigen erwachsenenpädagogischen Vorstellungen von Lehr-Lernkontexten und an Normalitätserwartungen an die Beschaffenheit von Lernorten und dortigen Abläufen, die sich qua ständiger Wiederholung zu objektivierten Mustern institutionalisieren und sich als kollektive Normierung möglicher Handlungsoptionen reproduzieren (Löw 2001, S. 168; S. 214 f.).

Gegenstand unserer Analyse ist insofern die Herstellung von Räumen im Handeln (ebd., S. 219), d. h. es geht primär um die Motive und Gestaltungskomponenten der professionell-pädagogischen Raumkonstitution. Die Folgen der Raumherstellungsprozesse, bspw. in Form von Angebotsprogrammen, sind eng mit dem Herstellungsprozess verwoben, analytisch hier jedoch nicht erfasst.

\section{Inklusionsarchitekturen: Zugriff und Rahmenstruktur des Modells}

Antworten in unserer Erhebung wie diese, ,dass die, die die Steuerung und die (...) Hoheit über die Räume haben, dass sie diesen Aspekt [die Inklusion; 
SSB/EF] bedenken, das ist schon mal das erste" (INT_7) ${ }^{1}$, rücken die Bedeutung der professionell Tätigen an institutionalisierten Lernorten in den Fokus. Der Forschungszugriff auf Wahrnehmung und Vorstellungen von Raum und auf das räumliche Handeln von Professionellen, konkret: ihre pädagogisch-professionellen Umsetzungsverfahren und -logiken an Lernorten, eröffnet Erkenntnisse über (An-)Ordnungen inklusiver Lernorte und das Spannungsgefüge ihrer hegemonialen Räumlichkeit (Kessl und Reutlinger 2010b), wie mit dem entwickelten Modell der Inklusionsarchitekturen und der folgenden Detailanalyse zu zeigen ist.

\subsection{Methodologische Rahmung und methodisches Verfahren}

Während die Anerkennung einer je ,unermesslichen“ individuellen Raumperspektive unaufhebbar bleibt (Löw 2001, S. 220; Ebner von Eschenbach 2017), unterstellen wir methodologisch Professionellen die Fähigkeit, Herstellungsprozesse sozialer Räume erklären und darstellen zu können (ebd.). Für diese Darstellungen war für uns weiterführend, dass sich mit Löw das raumkonstituierende Handeln der professionell Tätigen mit dem prozessunterbrechenden (Zwischen-)Ergebnis einer je spezifischen „,relationalen (An)Ordnung sozialer Güter und Menschen (Lebewesen) an Orten “ (Löw 2001, S. 224; Herv. i. Orig.) analytisch unterteilen lässt in zwei sich gegenseitig bedingende Prozesse: in Spacing und Syntheseleistung (ebd., S. $158 \mathrm{ff}$.). Spacing bezeichnet den Akt des Platzierens bzw. das Platziertsein von sozialen Gütern und Menschen an Orten. Diese über Platzierungen geschaffene (An)Ordnung wird allerdings erst in dem Moment der Syntheseleistung als Raum wirksam, wenn Menschen die Elemente der (An)Ordnung verknüpfen und zwar über die je individuellen Wahrnehmungs-, Vorstellungs- und/oder Erinnerungsprozesse.

Solche je individuell getätigten und professionell-pädagogisch legitimierten Syntheseleistungen sind das entscheidende Moment in der Wahrnehmungs- und vor allem auch Imaginationsleistung von den materiellen, soziostrukturellen und symbolischen Gegebenheiten im Sinne von (An)Ordnungen eines Lernorts. Professionell getätigte Syntheseleistungen entscheiden nicht nur über Einschließungen und Ausschließungen an bzw. von Lernorten, sondern, so Löw, über die jeweils getätigten Verknüpfungen werden auch die Dimensionen von Rang und Assoziation wirkmächtig repetitiv institutionalisiert und reproduziert; folglich ist „,bei institutionalisierten Verknüpfungen die synthetisierende Person oder Gruppe für die Reproduktion sozialer Ungleichheit von entscheidender Bedeutung“ (Löw 2001, S. 215). So werden bspw. bauliche Barrieren eines Lernorts als unüberwindbares bzw. als überwindbares Hindernis für inklusive Angebote wahrgenommen, professionell-pädagogisch legitimiert und (nicht) als Handlungsauftrag an den Lernort und die Konstituierung des pädagogischen Lernraumes imaginiert. Die planend-disponierenden Tätigkeiten der professionell Tätigen vollziehen sich zum Teil als normiertes Spacing und als nichtwillkürliche Syntheseleistungen (s. Kap. 2.2), doch bleiben die vorgängigen Normierungen der sozialen Raumpraktiken grundsätzlich verhandelbar und kontingent, können also in Planung und Performanz eigene Regeln entwickeln und Ambivalen-

\footnotetext{
1 Aus Gründen der Lesefreundlichkeit wird auf die detaillierte Angabe der Sequenzen aus den zu allen Interviews vorliegenden Transkriptionen verzichtet; die Nummer verweist auf das jeweilige Interview.
} 
zen oder Gegenstrukturen entfalten (Rau 2013, S. 183). Derart kondensieren sich in den Syntheseleistungen graduell unterschiedliche Varianten eines professionellen Gestaltungsoptimismus (Fritsche 2010) bzw. -pessimismus gegenüber einer Operationalisierung von Inklusion.

Methodisch haben wir in der Datenerhebung leitfadengestützte, theoriegenerierende Experteninterviews (Bogner et al. 2014) genutzt. Identifiziert sind jene als Expertinnen und Experten, die ,mit ihren Deutungen das konkrete Handlungsfeld sinnhaft und handlungsleitend für andere (...) strukturieren“" (ebd., S. 13; Herv. i. Orig.), in diesem Falle den Zugang und das Angebot für erwachsene Lerninteressierte an intentionalen und institutionalisierten Lernorten im öffentlichen Raum. Hierzu wurde ein kontrastives Sample von planend-disponierend Tätigen an explizit als inklusiv deklarierten Lernorten erhoben ( $n=7$; Datenerhebung erfolgte Ende 2014/ Anfang 2015). Die Kriterien der Kontrastierung waren die disziplinär-professionelle Herkunft der Tätigen (Erwachsenenbildung bzw. Behindertenpädagogik) und das Praxisfeld ihrer hauptamtlichen Tätigkeit (Lernort der allgemeinen Erwachsenenbildung (Bsp. Volkshochschule bzw. Lernort eines Trägers der Behindertenhilfe), um den disziplinär-professionellen Überschneidungsbereichen bei der Operationalisierung von Inklusion gerecht zu werden und für die Erkenntnisgewinnung zu nutzen. Es entstand ein Sample von sieben hauptamtlich tätigen Personen, von denen vier an Lernorten der allgemeinen Erwachsenenbildung, zwei an Lernorten von Trägern der Behindertenhilfe und eine primär im akademischen Feld tätig waren; territorial bezog das Sample drei norddeutsche Regionen ein. Die Datenauswertung folgte dem iterativen Auswertungsprozess der Grounded Theory (im Anschluss an Strauss und Corbin 1996; Strübing 2014; Codierung mithilfe von MAXQDA). Es galt, über die Herausarbeitung von Kategorien einen heuristischen Rahmen für eine empirisch fundierte Theorie als Erklärungsmodell für Varianz in der und Konsequenzen von der Operationalisierung von Inklusion an Lernorten als Akt der Herstellung sozialer Räume im und durch das Handeln von professionell Tätigen zu entwickeln. Diesen heuristischen Rahmen (das Modell der Inklusionsarchitektur) gilt es durch Anschlussforschung in Gestalt von partizipativer Forschung mit Menschen mit Lernschwierigkeiten (vgl. z. B. Buchner et al. 2016) weiterzuentwickeln und die Reichweite der Erhebung mit der Subjektperspektive von Lerninteressierten auf inklusive Lernorte zu vergrößern.

\subsection{Ergebnis der Analyse: Rahmenstruktur des Modells der Inklusionsarchitekturen}

Für die geleistete Systematisierung von professionellen (An)Ordnungen inklusiver Lernorte nehmen wir mit dem Begriff der Inklusionsarchitektur Anschluss an Knolls Ausführungen zu „Lernarchitektur“: ,,jene Baukunst, die dem Lernen dient oder dienen soll, indem sie hierfür Häuser entwirft und verwirklicht, Räume ausstattet, Plätze inszeniert (...) um sich Wissen anzueignen, Fähigkeiten zu erweitern, die eigene Person weiterzuentwickeln, sich zu bilden“ (1999, S. 24). Diese Verschränkung von Materialität, professioneller Inszenierung und der Intentionalität von Lernen und Bildung ist anschlussfähig an unser Verständnis inklusiver Lernorte und -räume und 


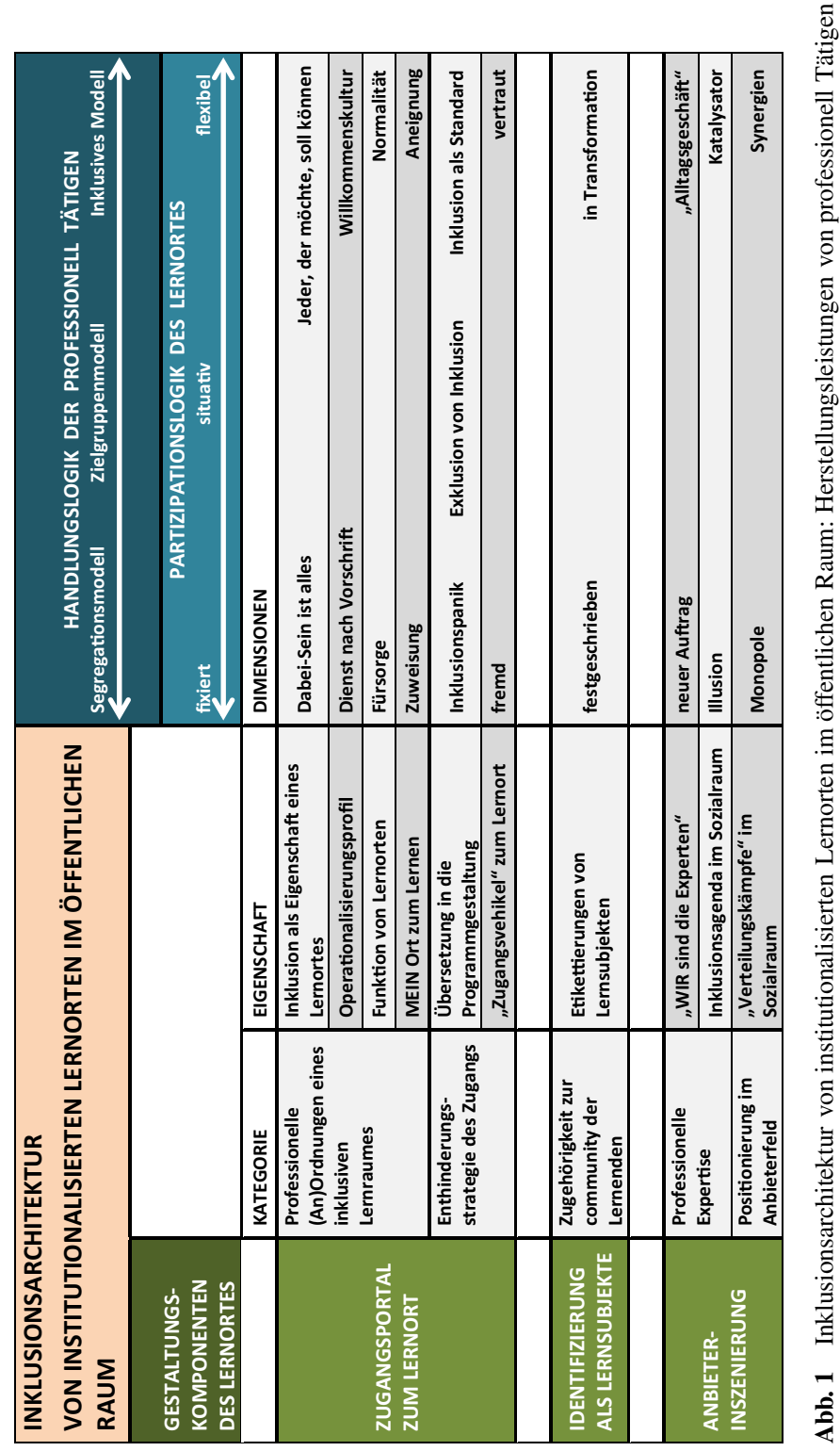


an unsere Perspektive der Herstellung sozialer Räume im und durch das Handeln professionell Tätiger.

Als Ergebnis des iterativen Auswertungsprozesses der Grounded Theory (s. oben) konnten Kategorien verdichtend identifiziert werden, die das zu untersuchende Phänomen (Operationalisierung von Inklusion) erklären. Jede Kategorie verfügt über Eigenschaft(en), die qualitativ entlang von Dimensionen beschrieben werden können und sich aus der empirisch vorgefundenen Varianz der Aussagen generiert haben. Das so entwickelte Modell von Inklusionsarchitekturen von institutionalisierten Lernorten im öffentlichen Raum (s. Abb. 1) $)^{2}$ identifiziert die ermittelte spezifische Variationsbreite räumlichen Handelns zwischen Determinierung und Eigendynamik in Bezug auf die Frage, wie ein inklusiver Raum des Lehrens und Lernens an dem je konkreten Lernort von professionell Tätigen hergestellt wird (s. Kap. 3.2.1). Zudem bringt die Analyse der Verknüpfungen der materiellen, soziostrukturellen und symbolischen Dimensionen des räumlichen Handelns und entstehender (An)Ordnungen im Ergebnis zwei zentrale, miteinander verknüpfte Logiken hervor: eine Handlungslogik der Professionellen und eine Partizipationslogik des Lernortes (s. Kap. 3.2.2).

\subsubsection{Gestaltungskomponenten des Lernortes}

Zunächst kategorisiert das Modell die Aussagen der professionell Tätigen zu Spacing und Syntheseleistungen in Bezug auf inklusives Lehren und Lernen am Lernort. Erkenntnisgenerierend waren sowohl Aussagen über eigenes Nicht-/Handeln im professionellen Kontext als auch Aussagen über das Nicht-/Handeln anderer Personen in vergleichbaren Kontexten - wenn dies genutzt wurde als Referenzfolie für die Erläuterung des eigenen Operationalisierungsverständnisses oder Handelns. Angestrebt wurde im Forschungsdesign also nicht eine externe Evaluierung von de facto räumlichem Handeln, sondern wesentlich war die Erhebung der von den Professionellen je genutzten materialen, soziostrukturellen und symbolischen Elemente in Spacing und Syntheseleistungen und damit Verstehenszugänge auf Prozesse der Herstellung und Veränderung sozialer Räume.

Die Aussagen lassen sich verdichten zu drei Gestaltungskomponenten von professionell Tätigen für das Ziel einer Operationalisierung von Inklusion an einem Lernort:

- Zugangsportal zum Lernort als Kerngestaltungskomponente, die am wirkmächtigen Scharnier des Zugangs zum Lernort arbeitet - wem wird welche Art von $\mathrm{Zu}$ gang zum Lernort professionell zuerkannt und de facto ermöglicht, welche Plätze werden am Lernort wem zugewiesen?

- Identifizierung als Lernsubjekte - wer gilt als Adressatin und Adressat von Angeboten der Erwachsenenbildung und wer wird als welche Art von Klientel durch den Anbieter identifiziert?

- Anbieterinszenierung - wie inszenieren sich die Anbieter im Angebotsfeld und wer deklariert wen als Experte für Inklusion?

\footnotetext{
2 In der Grafik sind mit Anführungszeichen sog. In-vivo-Codes gekennzeichnet, d. h. Wörter, die die Interviewten selbst gebraucht haben. Großschreibung dient dem Hinweis auf eine Betonung.
} 


\subsubsection{Handlungslogik der professionell Tätigen-Partizipationslogik des Lernortes}

Jene Herstellungsleistungen von inklusiven Räumen lassen zwei Logiken erkennbar werden, die die je spezifische Variationsbreite in den Aussagen der professionell Tätigen als eine Dimensionalisierung zwischen zwei Polen abbildet: Erstens ist eine übergeordnete Handlungslogik der professionell Tätigen zu identifizieren, die auf bestimmte Umsetzungsmodelle verweist und darin zwischen den Formen Segregationsmodell - Zielgruppenmodell - inklusives Modell variiert. Diese Unterteilung ist nicht trennscharf, sie zeigt aber die Tendenz der Operationalisierung an. Parallel hierzu verläuft, zweitens, eine Partizipationslogik, die über räumliches Handeln eröffnet wird und sich am Lernort materialisiert. Erkennbar werden in den Dimensionalisierungen der Partizipationslogik unterschiedliche Qualitäten von operationalisierter bzw. imaginierter Partizipation:

- Ein Segregationsmodell entspricht einer fixierten Qualität von Partizipation, der zufolge von den professionell Tätigen un-/bewusst, mehr oder weniger explizit bestimmte Lernende zu je bestimmten Orten, Lernzielen und/oder Merkmalen nach einem weiterhin territorial segregierend arbeitenden Paradigma zugeordnet werden.

- Im Zielgruppenmodell lösen sich diese starren Vorstellungen auf hin zu einer situativen Qualität von Partizipation, d. h. zu situativ gefällten Entscheidungen im Planungs- und Organisationsprozess in Bezug auf einzelne Lernende, Lerninteressierte und/oder Handlungserfordernisse. Gleichwohl bleiben die Personen weiterhin einer bestimmten, professionell definierten Gruppe von Lernenden zugeordnet.

- Das inklusive Modell geht über situative Einzelfallentscheidungen und vorgängige Zuordnungen transsektional hinaus und sieht als Qualität von Partizipation an einem Lernort eine flexible Struktur vor. Sie strebt strukturell eine inklusionsförderliche Gestaltung des gesamten Lernorts an und eröffnet damit Flexibilität für einen weiten Möglichkeitsraum von Partizipation, für eine Identifizierung als Lernsubjekt, für professionelle Annahmen von Lernzielen und -interessen oder auch für ein (unkonventionelles) Finden von Lösungen für Handlungsnotwendigkeiten im institutionellen Alltag.

Um einen Einblick in die Ergebnisse und damit Verstehenszugänge auf das räumliche Handeln und auf Spacing und Syntheseleistungen der Professionellen sowie der daraus resultierenden sozialen Räume des Lehrens und Lernens zu geben, werden die Pole der Dimensionalisierungen erläutert. Als Fokus der Detailanalyse ist die Kerngestaltungskomponente ausgewählt.

\section{Detailanalyse - Zugangsportal zum Lernort als Kerngestaltungskomponente der professionell Tätigen}

Die Kerngestaltungskomponente arbeitet am wirkmächtigen Scharnier des Zugangs zum Lernort: Wem wird welche Art von Zugang zum Lernort professionell zuerkannt und de facto ermöglicht? Zugang stellt ein vorgelagertes Entscheidungsmoment zum 
Ziel der Operationalisierung von Inklusion dar: „,Bevor irgendjemand in irgendeinem Kurs sitzt, haben wir unsere Arbeit ja eigentlich schon gemacht“ (INT_7). Zugang wird verhandelt über die Metaebene der Imagination eines inklusiven Raums des Lehrens und Lernens (s. Kap. 4.1 Professionelle (An)Ordnungen eines inklusiven Lernraums) sowie über praktische Umsetzungsmechanismen (s. Kap. 4.2 Enthinderungsstrategie des Zugangs).

\subsection{Professionelle (An)Ordnungen eines inklusiven Lernraums}

Solche (An)Ordnungen werden in den Aussagen zum Raumverständnis und zum räumlichen Handeln der professionell Tätigen als Ensembles aus materiellen, symbolischen und soziostrukturellen Elementen sichtbar und lassen vier Eigenschaften erkennen.

\subsubsection{Inklusion als Eigenschaft eines Lernorts: zwischen Dabei-Sein ist alles und Jeder, der möchte, soll können}

Das Raumverständnis als Rahmung zu Prozessen räumlichen Handelns variiert zwischen einer Priorisierung von Territorialität/Materialität als Inklusion bis hin zur Zuerkennung von größtmöglicher Autonomie in Entscheidungsprozessen an lerninteressierte Subjekte.

Bei Dabei-Sein ist alles folgen die Handlungslogiken dem traditionellen Segregationsmodell und sehen fixierte (An)Ordnungen von sozialen Gütern und Lebewesen an Orten vor. Das Label Inklusion wird instrumentalisiert: Als „Kulisse“ (INT_7) einer Institution, eine Art Potemkin'sches Dorf, das territorial Zugang eröffnet, aber in Beibehaltung der Segregationslinien und in einer Funktionalisierung für andere (zum Wohle der Außenwirkung , lassen wir da ein paar durch das Bild rollern oder rumpeln ... “ (INT_7) bzw. „da schiebe ich dann irgendwie noch drei Rollstühle mit rein und das ist auch schön gewesen für die anderen“ (INT_5)). Körper - in diesem Falle Menschen mit Behinderungen - werden bewusst an bestimmten Orten innerhalb der Institution platziert, unabhängig davon, ob Lernprozesse angeregt werden oder Lernziele vorgesehen sind (INT_2; INT_7; INT_6), und ob das territoriale Dabei-Sein atmosphärisch in eine positive Gruppenerfahrung mündet oder sich Exklusion nunmehr als negativer Erfahrungswert in die Kurse selbst verschiebt (INT_4). Professionalität bleibt in dieser Partizipationslogik in fixierten, sprich üblichen Bahnen (als „Konsens-Suppe“, man würde sich „im Gutmenschentum wohnlich einrichten“; INT_5). Es werden barrierearme Zugänge als hilfreich wahrgenommen - allerdings für den Normalbetrieb (so diene ein Fahrstuhl v. a. dem Transport von Lasten; INT_7).

Für die Bewegung hin zum anderen Pol der Eigenschaft, zu Jeder, der möchte, soll können, ist das Ausmaß der Zuerkennung größtmöglicher Autonomie in Entscheidungen maßgeblich. Inklusion wird imaginiert als Gleichstellung mit dem sog. „Normalbürger“ (INT_2) und dem Prinzip der Freiwilligkeit von Teilnahme oder eben auch Nicht-Teilnahme als Folge autonomer Entscheidungen und individueller Erfahrungen (INT_7; INT_4): „Jeder kann an diesem Ort das lernen, was er sich irgendwie vorgenommen hat" (INT_5). Ein solch inklusives Modell in der 
Handlungslogik basiert auf einer flexiblen Partizipationslogik, die nicht pauschal „für jeden das Gleiche“ einfordert, sondern ,für jeden das Passende“ (INT_7) und diesem professionell assistiert (INT_4; INT_1; INT_2). Räumlichem Handeln der Professionellen wird eine Revision von Rollenzuweisungen und (An)Ordnungen am Lernort zugewiesen. Genannt wird, hierzu Bedarfe und Bedürfnisse von Lerninteressierten vorgängig abzufragen (INT_3; INT_4) und den Expertenstatus auf diejenigen zu verlagern (hier: Menschen mit Behinderungen), die eine Beurteilung des Status quo des Lernorts tatsächlich vornehmen können (,,das seht ihr gar nicht, aber das ist eigentlich total bescheuert" (INT_7)).

\subsubsection{Operationalisierungsprofil: zwischen Dienst nach Vorschrift und Willkommenskultur}

Auf der programmatisch-strategischen Ebene der Operationalisierung von Inklusion am Lernort findet sich in den Aussagen eine Unterteilung in reaktive Handlungsmuster in Form von operativen Minimallösungen bis hin $\mathrm{zu}$ einem umfassenden Inklusions-Mainstreaming.

Dienst nach Vorschrift bezieht sich auf ein Handeln, das die bildungspolitische Agenda als ,Inklusionsstress“ (INT_7) wahrnimmt. Eine von extern geforderte Operationalisierung beschränkt Inklusion in der Handlungslogik auf sichtbare Nachweise am Lernort, sei es durch Körper (Teilnehmende mit Behinderungen (INT_2)), durch Ausweisung einzelner Angebote im Programm (INT_7) oder durch Materialitäten (Fahrstühle, Rollstuhlrampen u. Ä.; s. z. B. INT_3). Räumliches Handeln in dieser Partizipationslogik arbeitet im engen Radius einer operativen Minimallösung, bei der inklusive bauliche Arrangements entstehen, aber ohne die Gesamtinstitution als Lernort wahrzunehmen (INT_5).

Kernmerkmal der Verschiebung in Richtung einer Willkommenskultur wird der Bereitschaft der Professionellen zuerkannt, Reflexionsprozesse über die Multidimensionalität von Inklusion und ihrer Operationalisierung zu befördern. Prozessstruktur und Zielvision ist ein Inklusions-Mainstreaming am Lernort und zwar über die Dimension dis/ability hinaus. Als professionell Tätige gehe man vom einzelnen Kursort, „vom Raum hoch und alle Beteiligten müssen in dem Prozess einbezogen werden, die um diese Räume herumstehen und mit diesen Räumen was machen" (INT_7) (INT_3). Ein solches Operationalisierungsprofil wird als strudel- oder spiralförmiger Prozess beschrieben (INT_2; INT_7), der die Reflexion des gesamten Bedingungsgefüges der (An)Ordnungen am Lernort zum Ziel einer kontinuierlichen Qualitätsverbesserung interpretiert (INT_2; INT_4). Professionalität wird ausgedeutet als strategischer Abbau von Barrieren im Zugang, in der Vermittlung und in den Köpfen aller Beteiligten (INT_2; INT_5). Der Mehrwert einer solchen Partizipationslogik liegt im Alltag der Institution, über dis/ability hinaus auf verschiedenste Formen sozialer Ungleichheit bei Lerninteressierten flexibel reagieren zu können (INT_7) - und zugleich professionelles Grenzbewusstsein transparent zu machen: „Gut, ich nenne es Inklusion, weiß aber, dass es eigentlich noch nicht Inklusion ist“ (INT_1). Willkommenskultur meint dann, in z. B. den Programmankündigungen ,InklusionsSIGNALE“" (INT_7) über fehlende räumliche Barrierefreiheit zu setzen, also sichtbar zu artikulieren, dass hier (noch) ein Mangel besteht. 


\subsubsection{Funktion von Lernorten: zwischen Fürsorge und Normalität}

Die Forderung nach inklusiven Lernorten stellt zur Debatte, welche Funktion ein Lernort übernimmt bzw. übernehmen sollte, sowohl als Teil eines erwachsenenpädagogischen Professionalitätsverständnisses als auch als Teil gesellschaftlicher Transformation. Grundmerkmal ist das Ausmaß der Irritation von Normalitätserwartungen: zwischen der Bewahrung von unterstellter Homogenität bis hin zur Zumutung einer anderen Normalität.

Inklusion als Reproduktion des Segregationsmodells wird in den Aussagen eine paternalistisch-fürsorgende Funktion eines Schutzraums für Menschen mit Behinderungen zugeschrieben, die je spezifische Plätze, Erwartungen und Angebote vorsieht. Solch ein „Soziotop“ (INT_5) oder „Ghetto“ (INT_7) bietet atmosphärisch sozialen Schutz und vergleichbare Lebens- und Lernkontexte unter den Teilnehmenden (INT_6). Eine Revision der (An)Ordnung ist weder in der Handlungs- noch in der Partizipationslogik das Ziel; Menschen mit Behinderungen werden außerhalb des öffentlichen Raumes „,bedient“ und dort belassen (INT_4).

In Richtung des anderen Pols verschiebt sich die Funktion eines Lernorts im Sinne des Erlebens und Anerkennens eines inklusiven Lernorts als Normalität, nicht Ausnahme: wenn „DAS zur Normalität wird, dass man sich in den Räumen begegnet, sich wahrnimmt und das gewahr wird, da gibt es Menschen, die sind so und so und so, und ich bin SO, und wir sind alle hier" (INT_4). Deutlich wird in den Aussagen, dass eine Revision traditioneller (An)Ordnungen keineswegs konflikt- oder spannungsfrei verläuft; Dynamik, Reibungen, Irritationen im institutionellen Alltag werden als zwar ,anstrengend“, jedoch notwendig für institutionelle wie personale Weiterentwicklung interpretiert (INT_7; INT_1; INT_4). Diese veränderte Handlungslogik allen Beteiligten und Ebenen „zuzumuten“ (INT_7) wird als Aufgabe der professionell Tätigen benannt: als Institution auch Irritationen bei Kursteilnehmenden ohne Behinderungen in Kauf zu nehmen (INT_4), eine andere Ästhetik von Körpern am Lernort zuzulassen (INT_1) oder institutionelle Abläufe wie bspw. Anmeldevorgänge auch Menschen mit Behinderungen zuzutrauen (also Schonräume bewusst vorzuenthalten), gleichwohl dies institutionelle Prozeduren verlangsame (INT_7). Die Partizipationslogik meint dann Normalisierung durch „Sensibilisierung “ und „Desensibilisierung “, letzteres v. a. der Menschen ohne Behinderungen: ,gewöhnt euch einfach dran! “ (INT_7).

\subsubsection{Mein Ort zum Lernen: zwischen Zuweisung und Aneignung}

Für Zugang sind neben infrastrukturellen Rahmungen zugleich die in Räumen eingelagerten Fremd- und Selbstzuschreibungen entscheidend, welcher Ort für wen zum Lernen gedacht und vorgesehen ist. Die Aussagen verweisen auf zwei Pole, auf Zuweisung und Aneignung.

Im Segregationsmodell ist die territoriale Zuweisung von Körpern an Plätzen und Orten ein fixiertes, von außen definiertes Konstrukt, das für Lerninteressierte mit Behinderungen eine passive Rolle und die Wahrnehmung eines Zugangs auch zu anderen Plätzen und Orten nicht zwingend vorsieht: „, Wir kommen aus einer jahrzehntelang eh ehm andauernden segregierenden Verfahrensweise mit diesen 
Menschen und mit denen ist wirklich verfahren worden. Das heißt, deren Selbstständigkeit schon allein in der, in der eh Wahrnehmung fremder Räume ist ja nicht so ohne Weiteres gegeben“ (INT_2). Dieser Zuweisungscharakter hebt sich nicht automatisch auf, wenn es sich um einen als inklusiv deklarierten Lernort handelt (INT_5). Zur Aushandlung steht weiterhin der Status am Lernort, also ob Lerninteressierte mit Behinderungen bspw. als reguläre Klientel von Lernangeboten gesehen werden oder als (unbezahltes) Hilfspersonal für einfache Tätigkeiten im Tagesgeschäft eines Lernorts (Flyer verteilen u. Ä.; INT_6). Gleiches wird für die Kurswahl genannt, die dann vorrangig über Angehörige oder Betreuungspersonen erfolgt (,wenn dort äh die Betreuer sagen, Mensch das ist doch mal 'ne schöne Sache. Und dann werden sie auch dort angemeldet" (INT_6)).

Mit der Verschiebung zum Pol Aneignung gerät der Zuweisungscharakter in Bewegung, was eine Irritation der Machtstrukturen, der „Menschen, die diesen Raum quasi beherrschen“ (INT_7), darstellt. Es geht um Aneignung nicht irgendeines, sondern eines bestimmten Orts im öffentlichen Raum: „So oh wow, irgendwie geh auch (.) ich mache auch so einen Kurs. Und das ist wirklich beeindruckend. Also ICH mache so einen Kurs. (...) Sonst habe ich das quasi immer nur wahrgenommen, dass hinter geschlossenen Türen irgendwas passiert und jetzt bin ich Teil" (INT_5). Die physische Okkupation eines Orts befördert positive Emotionen wie Stolz (INT_7) und macht den Ort zu meinem Lernort als Teil des regulären, nicht des Sondersystems (,,also allein schon in so ein Haus, so ein Haus zu betreten und zu sehen, da gehöre ich auch hin “ (INT_7) (INT_4; INT_2).

\subsection{Enthinderungsstrategie des Zugangs}

Neben der Metaebene der Imagination eines inklusiven Raums sind für die Operationalisierung von Inklusion gleichfalls praktische Umsetzungsmechanismen von Bedeutung. Der Praxistransfer richtet sich in den Aussagen auf Aspekte der Programmgestaltung sowie auf Gelegenheitsstrukturen am und zum Lernort.

\subsection{1 Übersetzung in die Programmgestaltung: zwischen Inklusionspanik über Exklusion von Inklusion bis hin zu Inklusion als Standard}

Das Handeln zur Operationalisierung von Inklusion materialisiert sich im Programmprofil der Institution, den Ankündigungstexten oder auch im Layout. Programmgestaltung als planend-disponierendes Handeln lässt sich zuordnen zwischen den Polen von Inklusionspanik über Exklusion von Inklusion bis hin zu Inklusion als Standard.

Die Aufforderung zur Operationalisierung kann Inklusionspanik erzeugen und eine Handlungslogik von Nicht-Handeln als Abwehr. Allein die Imagination einer Transformation der (An)Ordnungen rufe emotionale Bedenken bei den am Lernort professionell Tätigen hervor, eine „Antizipation von Schwierigkeiten“ (INT_7), ,,irrationale Ängste“ (INT_2) - und dies noch bevor irgendeine Transformation eingesetzt hat. Inklusion wird als Worst-case-Szenario imaginiert, in Bezug auf zusätzliche Aufgaben und Kosten, bspw. durch kostenfreie Begleitung durch Assistenzpersonen (INT_7; INT_2), auf eine Überforderung der Kursleitenden (INT_4; INT_6), auf Fragen des Umgangs mit Hilfsmitteln am Lernort (Bsp. Blindenhund) oder auf die 
antizipierte Anspruchshaltung der neuen Klientel: „man stellt sich dann den völlig hilflosen schwerstmehrfachbehinderten Menschen vor, der plötzlich vor der Tür steht und ein ehm hohes Niveau des Englischkurses sich einklagen will“" (INT_2). Die Partizipationslogik am Lernort bleibt fixiert, um Irritationen vorzubeugen (INT_7).

Im Zuge der Operationalisierung von Inklusion änderten sich, so die Aussagen, Handlungs- und Partizipationslogiken, zunächst sporadisch und situativ in Form einer Exklusion von Inklusion in Sonderbereiche und -formate des Programms oder in Form von situativer Einzelinklusion von Lerninteressierten, die gebunden ist an die Aktivität bestimmter Professioneller. Inklusive Lehr-Lernsettings entstehen in dieser Logik z. B. über Einzelanfragen an Kursleitende durch Lerninteressierte mit Behinderungen (INT_6). Gründe gegen eine über das Situative hinausgehende Operationalisierung werden genannt mit Blick auf die Komplexität der Gestaltung von Ankündigungstexten in einfacher/leichter Sprache (INT_1; INT_4), auf fehlendes pädagogisches Know-how von Kursleitenden für inklusive Kurse (INT_6) oder auf höhere Kosten für inklusiv ausgebrachte Kurse. Inklusion wird zu einem Zielgruppenmodell für v. a. Lerninteressierte mit Lernschwierigkeiten konstruiert, das im regulären Programm erscheint, aber in Kooperation mit Trägern der Behindertenhilfe angeboten und z. T. in Gänze an die dortigen Lernorte territorial verlagert wird: Exklusion von Inklusion.

Die Kritik an einer Auslagerung (inklusive Kurse würden so ,im eigenen Haus ein Stück weit entsorgt“ (INT_7)) weist zum Pol von Inklusion als Standard. Weniger der Nachweis von Barrierefreiheit ist maßgeblich, sondern Handlungs- und Partizipationslogik spiegeln trotz anerkannter Herausforderungen einen Gestaltungsoptimismus wider: ,wir lassen jetzt mal da irgendwas ZU und gucken überhaupt wie das LÄUFT“ (INT_7). Das heiße nicht, Bedenken nicht ernst zu nehmen, sondern im Praxistransfer jeweils flexibel nach Lösungen zu suchen und dies in Bezug auf die lerninteressierte Person, nicht nach vorgängig definierten Zielgruppenkonzepten (INT_4). Für eine Person im Rollstuhl müsse nicht automatisch ein Kurskonzept umgestülpt werden; Kurse mit bestimmten Lernmethoden seien hingegen transparent anzukündigen (INT_4). Inklusion ist gelungen, wenn sie zum Standard wird (INT_7; INT_4; INT_5) und damit insgesamt einer Idee des Inklusions-Mainstreamings (s. oben) entspricht.

\subsection{2 „Zugangsvehikel“ zum Lernort: zwischen fremd und vertraut}

Auf der Ebene der Enthinderungsstrategie wird in den Aussagen deutlich, inwiefern materielle, soziostrukturelle als auch symbolische Komponenten für die Zugänglichkeit eines Lernorts im Sinne der Pole von fremd bzw. vertraut wirken und damit weniger bzw. mehr als „Zugangsvehikel“ zum Lernort fungieren können; an dieser Stelle tritt die Perspektive der Lerninteressierten selbst deutlicher in den Vordergrund (wenngleich durch die Linse der Professionellen).

Wenn der Zugang zum und der Lernort an sich von Lerninteressierten als fremd wahrgenommen wird, (re-)produziert dies Distanz. Dies entspricht einer Handlungslogik, die (An)Ordnungen am Lernort nicht reflektiert, sondern in ihrer bestehenden, fixierten Partizipationslogik belässt und damit ggf. Ausschließungen (re-)produziert (Aktbilder in einem Kursraum von Deutschsprachkursen befremden Lerninteres- 
sierte (INT_4), genauso wie die Schulatmosphäre von Kursräumen als Erinnerung an negative Schulerfahrungen (INT_3) oder die Einsparung der zwar „muffeligen Hausmeister" (INT_7), aber damit auch von Ansprechpersonen am Eingang von Häusern). Auf soziostruktureller Ebene wirken hohe Kosten für Kursangebote distanzierend oder anspruchsvolle Formatierungen von Programmheften, so dass nur Angehörige oder Betreuende, nicht aber Personen mit Lernschwierigkeiten diese lesen können (INT_3).

Eine Reduzierung der Befremdung wird benannt über verschiedene Arten von „Zugangsvehikeln“ (INT_3) zum Lernort, die die fixierte Partizipationslogik aufbrechen. Das „Warm“-werden mit einer Institution (INT_2) ist allerdings kein Automatismus, sondern von den Professionellen über systematische Angebote von Gelegenheitsstrukturen zu befördern: Über Schnupperangebote als Einstiegsniveau zu inklusiven Formaten und um Neugierde zu wecken (INT_2; INT_3) oder über informelle Gelegenheiten (gemeinsames Kaffeetrinken) als schrittweises territoriales Vertraut-werden mit dem Lernort und als Ort zur Weitergabe von Informationen (INT_6; INT_4). Inklusive Kursformate können zunächst einen Ort bieten, von dem weitere Schritte in das reguläre Angebot erfolgen können (INT_4). Dies gilt überdies für Menschen ohne Behinderungen, die als Begleitung oder Assistenz im „Kielwasser der Behinderten hineinschwimmen in die Kurse“ (INT_7), und derart auch vertraut werden mit inklusiven Lernorten.

\section{Identifizierung als Lernsubjekte und Anbieterinszenierung}

Neben der Kerngestaltungskomponente können im Modell der Inklusionsarchitektur zudem zwei weitere Gestaltungskomponenten des Handelns von professionell Tätigen für das Ziel einer Operationalisierung von Inklusion am Lernort identifiziert werden: Identifizierung als Lernsubjekte durch professionell Tätige sowie Anbieterinszenierung. Diese werden überblicksartig dargestellt.

\subsection{Identifizierung als Lernsubjekte}

Der Frage, wer als bildungsbedürftig und als bildungsfähig deklariert wird, kommt nicht nur beim Zugang zum Lernort, sondern auch in den Binnenstrukturen der LehrLernsettings eine hohe Bedeutung zu. Wer wird als zugehörig zur community der Lernenden identifiziert und mit welchen Etikettierungen belegt? Prozess und Inhalte solcher Etikettierungen werden beschrieben zwischen den Polen von festgeschrieben und in Transformation.

Etikettierungen von Lerninteressierten können in einer segregierenden Handlungslogik Zuschreibungsmuster und Normalitätserwartungen über Lernfähigkeiten oder -interessen (re-)produzieren und damit eine fixierte Partizipationslogik festschreiben. Dies gilt für alle: Lerninteressierte ohne Behinderungen werden als nichtzugehörig zur Gruppe derjenigen etikettiert, an die sich Kursankündigungen in leichter Sprache richten (INT_2), genauso wie Personen mit körperlichen Beeinträchtigungen per se mit der Unterstellung von Lernschwierigkeiten (INT_5) oder auch mit der Zuschreibung von Krankheit verbunden werden - was quer zur Eigen- 
wahrnehmung liegen kann (, Leute von außen würden sagen, ja, aber du bist doch so schwer krank, nä, das würde die Person aber jetzt gar nicht so werten, sondern sagen, ich fühle mich eigentlich ganz gesund" (INT_4)). Solche Etikettierungen geraten in Transformation über die Irritation von Normalitätserwartungen, nicht zuletzt durch das Erleben inklusiver Lehr-Lernsettings am Lernort (INT_1; INT_6). Die Partizipationslogik bricht auf, indem bspw. Kompetenz unvermutet zutage tritt: so in einem Kunstkurs (,Boah ok, eigentlich ist das, was jetzt die Frau mit Lernschwierigkeiten im Rollstuhl neben mir macht, eigentlich cooler" (INT_4)) oder bei einem Teilnehmenden mit Lernschwierigkeiten in einem Tanzkurs (,, er war der Star, weil, also er hat es sehr schnell umgesetzt, die ganzen Schrittfolgen, da hatten andere Teilnehmerinnen wirklich große Probleme“ (INT_4)). Das Verständnis von Kompetenz und Expertenstatus wird am Lernort neu definiert und dies wirkt auf das Handeln der professionell Tätigen. So treten Menschen mit Behinderungen nicht als Objekte von Fürsorge (s. oben) auf, sondern als bspw. Co-Kursleitende oder Evaluatoren für die Barrierefreiheit eines Lernorts (INT_5) und als hochkompetent für ihre Lebenssituation (,Eine hohe Kompetenz im Umgang mit eingeschränkten Möglichkeiten, auch irgendwie (---) halt, sich anzupassen an Scheißbedingungen“ (INT_5)).

\subsection{Anbieterinszenierung}

Ein Lernort ist Teil eines Sozialraums, in dem sich eine Vielzahl institutioneller Akteure befindet. In den Aussagen wird ein Ringen um professionelle Deutungshoheit und Ressourcen deutlich, das sich im räumlichen Handeln der Professionellen in den Kategorien Professionelle Expertise sowie Positionierung im Anbieterfeld widerspiegelt.

Erstere Kategorie bündelt Aussagen zu der Eigenschaft „WIR sind die Experten“ (INT_2). Konsens herrscht in der chronologischen Wahrnehmung, wonach erst über die Ratifizierung der UN-BRK die Fokussierung des Themas Inklusion auf die Kategorie dis/ability und im Besonderen auf Menschen mit Lernschwierigkeiten erfolgte. Wer vor diesem Hintergrund Experte für Inklusion sei, ruft ebenfalls Konsens hervor: Alle Träger sehen bei sich Expertenschaft für Inklusion - je nachdem, woraus Expertise abgeleitet wird (aus dem historischen Auftrag sozialer Inklusion von Erwachsenenbildung, aus behindertenpädagogischem Know-how, aus erwachsenenpädagogischem Wissen zu Planungs- und Vermittlungshandeln o. Ä.). Davon abhängig kann Inklusion im Fokus auf dis/ability sowohl als erwachsenenpädagogisches ,Alltagsgeschäft“ (INT_3) bewertet und an eine langjährig flexible Handlungs- und Partizipationslogik am Lernort geknüpft sein, als auch als ein neuer Auftrag wahrgenommen und mit einer eher fixierten Vorstellung einer nun spezifisch zu realisierenden Aufgabe verknüpft werden, die zunächst eher mit Inklusionspanik (s. oben) parallel läuft (gleiches gilt vice versa für die Ausdeutung von Inklusion als historischem Auftrag). Dieser Aspekt der professionellen Inszenierung gegenüber Inklusion wird ergänzt durch Aussagen zur Manifestierung der bildungspolitischen Inklusionsagenda im Sozialraum. Die Inklusionsagenda wird beschrieben zwischen den Polen von einer Illusion bis hin zu einem Katalysator. Illusion, weil in Bezug auf professionelles Handeln am Lernort Inklusion eher als Sparmaßnahme wahrgenommen wird (INT_3), nur langsam voranschreite und zwar Möglichkeiten eröffne, 
aber ohne strukturelle Unterfütterung mit Ressourcen stattfinde (,,dem traue ich auch nicht, diesen kleinen Schritten “(INT_5)). Kommt der Vergleich des Status quo des Themas Inklusion vor bzw. nach der Ratifizierung der UN-BRK als eine chronologische Komponente hinzu, verschiebt sich die Wahrnehmung hin zu einem Katalysator. Die UN-BRK habe „den Ball ins Rollen gebracht“ (INT_7) und eine hohe Aufmerksamkeit im Sozialraum für das Thema erwirkt, sei es in der Presse, in der kommunalen Verwaltung oder bei lokalen Unternehmen (INT_2; INT_6; INT_3; INT_7; INT_1). Dies habe neue Partizipationsmöglichkeiten für Lerninteressierte am Lernort und im gesamten Sozialraum erwirkt.

Die erhöhte Präsenz und Außenwirkung des Themas wird von den Professionellen andererseits mit Blick auf die eigene Positionierung im Anbieterfeld als Grund für „Verteilungskämpfe“ im Sozialraum angeführt. Eingeschätzt werden diese zwischen monopolistischen Vorstellungen in der Operationalisierung von Inklusion bis hin zum Leitmotiv von Kooperation als Synergien.

Monopolistische Vorstellungen, ein institutioneller „Belagerungszustand dieser Behinderten “ (INT_7), werden v. a. auf ökonomische Faktoren im Sozialraum bezogen angesichts begrenzter finanzieller Ressourcen für Schule, Erwachsenenbildung und Behindertenhilfe. „Kleine Pflänzchen“ (INT_3) der Kooperation könnten nicht darüber hinwegtäuschen, dass die öffentliche Aufmerksamkeit für Inklusion gleichzeitig die Ressourcenanteile der Träger schmälere (INT_5). Dies wirkt auf die professionelle Handlungs- und Partizipationslogik dahingehend, dass kein Träger die Hoheit über bestimmte Aufgaben oder Ressourcen verlieren möchte (INT_2; INT_5). Letztlich aber gehe dies zulasten der verschiedenen vulnerablen Gruppen in der Gesellschaft; es entstehen ,Verteilungskämpfe (...) wo eh schon nichts mehr, oder nicht mehr so viel zu holen ist" (INT_5). Im gleichen Zuge wird von den meisten Professionellen anerkannt, dass das Ziel der Operationalisierung von Inklusion und einer Breite von Partizipationsmöglichkeiten am Lernort Synergien im Sozialraum und in den Handlungslogiken am Lernort notwendig mache (INT_2; INT_1). Wenn man gemeinsam etwas ,wuppen“ (INT_5) möchte, muss sich die Perspektive wandeln von Konkurrenz zur Wahrnehmung komplementärer Expertise (INT_3). Eine Breite von Partizipationsmöglichkeiten wirkt positiv auf Lerninteresse und -gewohnheiten und nutze durch insgesamt erhöhte Teilnahmequoten letztlich allen Trägern.

\section{Schlussfolgerungen und Forschungsdesiderate}

Das Modell der Inklusionsarchitektur von institutionalisierten Lernorten im öffentlichen Raum bietet eine Matrix für die Weiterentwicklung zum einen der theoretischkonzeptionellen Begründungsdiskurse (Lindmeier und Lindmeier 2015) um Inklusion und der Anerkennung als ein mehrschichtiges pädagogisches Wirkungsgefüge aus Subjekten, Strukturen, Symbolik und Materialität; und zum anderen der Realisierungsdiskurse (ebd.) um Inklusion, um über pauschalisierende gut/schlecht, funktioniert/funktioniert-nicht-Bewertungen hinaus erwachsenenpädagogisch relevante Erkenntnisse zu den Konsequenzen räumlichen Handelns von professionell Tätigen für die Frage des Zugangs zu Lernorten und dortigen Partizipations(an)ordnungen zu bieten. Spacing und Syntheseleistungen der Professionellen (re-)produzieren nicht 
eine beliebige, sondern eine spezifische Varianz an inklusiven Lernorten. Erkennbar werden graduell unterschiedliche Varianten eines professionellen Gestaltungsoptimismus (Fritsche 2010) bzw. -pessimismus, welche wiederum die Bedeutung und Wirkmächtigkeit der synthetisierenden Personen, in diesem Falle der pädagogisch Tätigen, für die Reproduktion von sozialer Un-/Gleichheit im Zugang zum öffentlichen Raum des Lebenslangen Lernens hervorheben.

Mit Blick auf diese Frage der Reproduktion von sozialer Un-/Gleichheit möchten wir resümierend Löw zustimmen (s. Kap. 2.1), dass als Teil der relationalen Formen sozialer Ungleichheit im Zugang zu sozialen Gütern (Lernort) die assoziative Zugehörigkeitszuweisung von Lerninteressierten zu sozialen Gruppen eine machtvolle Wirkung entfaltet: Insbesondere die Zugehörigkeitszuweisung zur Gruppe der Menschen mit Lernschwierigkeiten, also Klassifikationen entlang kognitiver und/ oder körperlicher Performanz und Ästhetik, und der diesbezüglichen Operationalisierung von Inklusion wird durchweg als institutioneller Lackmustest benannt (und eher pessimistisch eingeschätzt). Deutlich wird hier, dass die Herstellung von Räumen im Handeln aufs Engste mit Körperlichkeit verbunden ist. (Re-)produktiv wirkt dies, da die Zuweisungen und (An)Ordnungen von Körpern durch Professionelle in Gestalt von Platzanweisern zumeist vollzogen werden gemäß von Zuschreibungen „an verkörperte Differenz als Mangel““ (Modes 2016, S. 184; vgl. auch Campbell 2009) - also defizitäre Körperlichkeit gleichgesetzt wird mit der Zuweisung der Mitgliedschaft bei einer als lernleistungsdefizitär etikettierten Zielgruppe.

An dieser Stelle plädieren wir zum Ziel der perspektivischen Ausdifferenzierung der erwachsenenpädagogischen Position zu Inklusion für die Rückbindung der Kategorie der Gleichheit nicht an personale Merkmale (z. B. kognitiver oder körperlicher Performanz, Ästhetik) oder an soziostrukturelle Ausgangsdispositionen der Lerninteressierten. Sondern wir sehen Gleichheit gegeben, im Anschluss an die Arbeiten von Fraser (1995), qua des individuellen Status eines jeden Lerninteressierten als Inhaber und Inhaberin von Menschen- und Bürgerrechten, d. h. dem Recht auf Bildung (United Nations 1948). Diesem Lerninteresse ist mit einem adäquaten Angebot zu begegnen - aufgrund des Rechts auf Bildung, nicht als Konsequenz der zugewiesenen Mitgliedschaft bei einer als defizitär etikettierten Zielgruppe. Auf der Grundlage dieser Gleichheit kann (Fähigkeit) und darf (Selbstbestimmung) jede und jeder sich entscheiden für eine Nicht-/Teilnahme, wie der sog. „Normalbürger“ (INT_2) auch (s. Kap. 4.1.1; vgl. Schreiber-Barsch 2016, 2017). Hier bleibt das Konzept der relationalen Zielgruppenbestimmung als Planungsprinzip in der Erwachsenenbildung von Schäffter (2014) zu prüfen, inwiefern sich eine selbstinterpretativ vorgenommene Ausweisung als Zielgruppe als Ausdruck einer Empowermentstrategie einer sozialen Gruppe (ebd., S. 35 f) perspektivisch als tragfähig erweisen kann.

Denn Zugang zu Lernorten und dortige Angebote sind unserer Meinung nach weniger an vorgängigen Zielgruppeneinteilungen zu orientieren, sondern basierend auf einer funktionalen Supportstruktur zu öffnen, d. h. einer pädagogisch-professionellen Unterstützung in der Funktion der Ermöglichung von Teilnahme und Lernen (wie bspw. Assistenzen, Team-Teaching, Lernhilfen, -materialien in einfacher/ leichter Sprache u. Ä.). Dies erfordert unseres Erachtens nicht zwingend ein Nochmehr an hochspezialisierter Fort- und Weiterbildung der erwachsenenpädagogischen Professionellen, sondern ein systematisches Zusammenführen vorhandener Experti- 
se aus Erwachsenenbildung, Behindertenpädagogik und Inklusiver Pädagogik sowie auf personaler Ebene der Professionellen eine Offenheit für Flexibilität im professionellen Denken und Handeln und für reflexives Erkennen vorhandener persönlicher Ressourcen für Diversität an Lernorten. Das Installieren einer solchen Supportstruktur wirkt über den Kontext von dis/ability hinaus inkludierend auf Lerninteressierte, bspw. auf solche mit anderer Herkunftssprache als deutsch, auf funktionale Analphabetinnen und Analphabeten, ältere Lerninteressierte usw. Was funktional im Kontext dis/ability ist, ist funktional für viele soziale Kategorien und trägt die Chance, Teilnahme insgesamt zu erhöhen.

Lernorte repräsentieren folglich ein territorial gerichtetes Brennglas auf ein abgrenzbares Innen und Außen im Sozialraum, auf einen alltäglichen Schauplatz der (konfliktären) Aushandlung von Bürgerrechten und der Anerkennung als zugehörig zur community der Lernenden; es ist ein Ort der Subjektkonstitution (Schroer und Wilde 2010, S. 182). Denn durch die pädagogisch-professionelle Bereitstellung solcher öffentlichen Räume und Plätze, an denen alltägliche Praktiken der Subjekte, wie bspw. Lernen, prozessiert werden können, ist ein institutionalisierter Lernort im Grunde eine politische Arena, in der politische Subjektivierungsprozesse qua Lernen, Handeln, Kommunizieren praktiziert werden (vgl. Biesta 2014; SchreiberBarsch 2017). Dies unterstreicht den handlungstheoretischen Impetus relationaler Raumkonzepte, wonach Lernorte eben nicht einfach Behälter von sozialen Gütern, Menschen und Ordnungen sind. Unterstellt werden die Prozesshaftigkeit und die Handlungsdynamik von und zwischen subjektiven wie kollektiven (An)Ordnungen, Machtstrukturen, professionellen Haltungen, Sinngebungs- und Handlungsmustern und dies in ihrer materiellen, soziostrukturellen und symbolischen Verwobenheit. Forschende Verstehenszugänge richten sich auf Strukturen und Gegenstrukturen (Rau 2013), auf das Gewollte und Nicht-Gewollte (Kessl und Reutlinger 2010b), auf Absichten und Nicht-Absichten (Reutlinger und Wigger 2010) in den Formationen des Lebenslangen Lernens: auf die hegemoniale Räumlichkeit (Kessl und Reutlinger 2010b).

Aus unserer Perspektive rückt dies die Kategorie der Aushandlung, eines negotiating (Berking et al. 2006), in den Mittelpunkt, auch als Forschungsdesiderat. Aushandlungen zwischen den verschiedenen Akteuren (Gesellschaft, Bürgerinnen und Bürgern, Institutionen, Kursleitenden, lerninteressierten Subjekten, Teilnehmenden), die sowohl horizontal als auch vertikal verlaufen, die mehr oder weniger offen sind, mehr oder weniger partizipativ, mehr oder weniger konfliktär. Der interaktionistische Ansatz von Strauss (1978) und sein Verhandlungsparadigma von sozialer Ordnung als einer ausgehandelten Ordnung könnten sich hier als weiterführend erweisen. Abschließend ist Hertel (2013) zuzustimmen, dass die Reproduktion sozialer Ungleichheit in Organisationen eingelagert ist - aber eben auch dort veränderbar (ebd., S. 166). Kessl und Reutlinger (2010b) fordern entsprechend eine „reflexive räumliche Haltung“ der professionell Tätigen ein, eine „systematische und möglichst umfassende Inblicknahme des jeweiligen räumlichen Erbringungszusammenhangs" (ebd., S. 251). Eine solche Inblicknahme kann über ein Modell wie das der Inklusionsarchitektur von Lernorten systematisierend angeregt und entlang der Mehrdimensionalität von Inklusion als einem vielschichtigen pädagogischen Wirkungsgefüge weiterführend für Theorie wie Praxis genutzt werden - nicht zuletzt, um die 
Gestaltungsmacht der professionell Tätigen in der Herstellung sozialer Räume für inklusives Lehren und Lernen herauszustellen.

Open Access Dieser Artikel wird unter der Creative Commons Namensnennung 4.0 International Lizenz (http://creativecommons.org/licenses/by/4.0/deed.de) veröffentlicht, welche die Nutzung, Vervielfältigung, Bearbeitung, Verbreitung und Wiedergabe in jeglichem Medium und Format erlaubt, sofern Sie den/die ursprünglichen Autor(en) und die Quelle ordnungsgemäß nennen, einen Link zur Creative Commons Lizenz beifügen und angeben, ob Änderungen vorgenommen wurden.

\section{Literatur}

Ackermann, K.-E. (2012). Zwischen den Stühlen. Erwachsenenbildung für Menschen mit geistigen Behinderungen. DIE Zeitschrift für Erwachsenenbildung, 2, 26-29.

Berking, H., Frank, S., Frers, L., Löw, M., Meier, L., Steets, S., \& Stoetzer, S. (2006). Negotiating Urban conflicts. Interaction, space and control. Bielefeld: transcript.

Bernhard, C., Kraus, K., Schreiber-Barsch, S., \& Stang, R. (Hrsg.). (2015). Erwachsenenbildung und Raum. Theoretische Perspektiven - professionelles Handeln - Rahmungen des Lernens. Bielefeld: wbv.

Biesta, G. (2014). Learning in public places: civic learning for the twenty-first century. In G. Biesta, M. De Bie \& D. Wildemeersch (Hrsg.), Civic learning, democratic citizenship and the public sphere (S. 1-11). Dordrecht: Springer.

BMAS - Bundesministerium für Arbeit und Soziales (2011). Unser Weg in eine inklusive Gesellschaft. Der Nationale Aktionsplan der Bundesregierung zur Umsetzung der UN-Behindertenrechtskonvention. Berlin: BMAS.

BMAS - Bundesministerium für Arbeit und Soziales (2016). „Unser Weg in eine inklusive Gesellschaft“. Nationaler Aktionsplan 2.0 der Bundesregierung zur UN-Behindertenrechtskonvention (UN-BRK). Berlin: BMAS.

Bogner, A., Littig, B., \& Menz, W. (2014). Interviews mit Experten. Eine praxisorientierte Einführung. Wiesbaden: VS.

Bremer, H., Kleemann-Göhring, M., \& Wagner, F. (2015). Weiterbildung und Weiterbildungsberatung für „Bildungsferne“. Bielefeld: wbv.

Buchner, T., Koenig, O., \& Schuppener, S. (2016). Inklusive Forschung. Gemeinsam mit Menschen mit Lernschwierigkeiten forschen. Bad Heilbrunn: Klinkhardt.

Burtscher, R., Ditschek, E. J., Ackermann, K.-E., Kil, M., \& Kronauer, M. (Hrsg.). (2013). Zugänge zu Inklusion. Erwachsenenbildung, Behindertenpädagogik und Soziologie im Dialog. Bielefeld: wbv.

Campbell, F. K. (2009). Contours of ableism. New York: Palgrave Macmillan.

Deinet, U. (2010). Aneignungsraum. In C. Reutlinger, C. Fritsche \& E. Lingg (Hrsg.), Raumwissenschaftliche Basics (S. 35-43). Wiesbaden: VS.

Der pädagogische Blick (2017). Raum und Räumlichkeit in pädagogischen Kontexten. Bd. 2.

DGfE - Deutsche Gesellschaft für Erziehungswissenschaft (2015). Inklusion als Herausforderung für die Erziehungswissenschaft. www.dgfe.de/fileadmin/OrdnerRedakteure/Stellungnahmen/2015_ Inklusion_Positionierung.pdf. Zugegriffen: 28. Juli 2017.

DIE Zeitschrift (2012). Erwachsenenbildung inklusive. Bd. 2.

Ebner von Eschenbach, M. (2017). Im Grenzbereich des Räumlichen. Vorüberlegungen zu einer topologischen Perspektive in der erwachsenenpädagogischen Raumforschung. Zeitschrift für Weiterbildungsforschung. https://doi.org/10.1007/s40955-017-0080-6.

Franz, J. (2016). Zur Bedeutung von „Raum“ in Organisationen allgemeiner Erwachsenenbildung - Ergebnisse einer qualitativ-rekonstruktiven Studie. Zeitschrift für Erziehungswissenschaft. https://doi. org/10.1007/s11618-015-0666-y.

Fraser, N. (1995). From redistribution to recognition. New Left Review, 212, 68-92.

Frey, O. (2004). Urbane öffentliche Räume als Aneignungsräume. Lernorte eines konkreten Urbanismus? In U. Deinet \& C. Reutlinger (Hrsg.), „Aneignung “ als Bildungskonzept der Sozialpädagogik (S. 219-233). Wiesbaden: VS.

Fritsche, C. (2010). Maßnahmen gegen Unordnungen im öffentlichen Raum - ein Systematisierungsversuch unterschiedlicher Strömungen. In C. Reutlinger \& A. Wigger (Hrsg.), Transdisziplinäre Sozialraumarbeit: Grundlegungen und Perspektiven des St. Galler Modells zur Gestaltung des Sozialraums (S. 55-67). Berlin: Frank \& Timme. 
Haar, G. (2014). Inklusion in der Einrichtung umsetzen. DIE Zeitschrift für Erwachsenenbildung, 4, 46-48. HBV - Hessische Blätter für Volksbildung (2015). Erwachsenenbildung und Inklusion. Bd. 4.

Heimlich, U., \& Behr, I. (2009). Inklusion von Menschen mit Behinderung in der Erwachsenenbildung/ Weiterbildung. In R. Tippelt \& A. v. Hippel (Hrsg.), Handbuch Erwachsenenbildung/Weiterbildung (3. Aufl. S. 813-826). Wiesbaden: VS.

Hertel, S. (2013). Das Inklusionspotenzial interorganisationaler Kooperationen. In K. Dollhausen, T.C. Feld \& W. Seitter (Hrsg.), Erwachsenenpädagogische Kooperations- und Netzwerkforschung (S. 157-170). Wiesbaden: Springer.

Hinz, A. (2013). Inklusion - von der Unkenntnis zur Unkenntlichkeit!? Kritische Anmerkungen zu einem Jahrzehnt Diskurs über schulische Inklusion in Deutschland. Zeitschrift für Inklusion-online.net, 1. www.inklusion-online.net/index.php/inklusion-online/article/view/26/26. Zugegriffen: 28. Juli 2017.

Kade, J., Nittel, D., \& Seitter, W. (2007). Einführung in die Erwachsenenbildung/Weiterbildung (2. Aufl.). Stuttgart: Kohlhammer.

Kessl, F. (2016). Erziehungswissenschaftliche Forschung zu Raum und Räumlichkeit. Eine Verortung des Thementeils „Raum und Räumlichkeit in der erziehungswissenschaftlichen Forschung“. Zeitschrift für Pädagogik, 1, 5-19.

Kessl, F., \& Reutlinger, C. (2010a). Sozialraum. Eine Einführung (2. Aufl.). Wiesbaden: VS.

Kessl, F., \& Reutlinger, C. (2010b). Sozialraum. In C. Reutlinger, C. Fritsche \& E. Lingg (Hrsg.), Raumwissenschaftliche Basics (S. 247-255). Wiesbaden: VS.

Knoll, J. (1999). Lernen im geschaffenen Raum: Zum materiellen Verständnis des Begriffs der „Lernarchitektur". DIE Zeitschrift für Erwachsenenbildung, 6, 24-25.

Köpfer, A. (2014). Raum und Stigma. Eine raumtheoretische Annäherung an die kritische Rolle von Integrationshelfer/innen in inklusiven Settings. VHN. https://doi.org/10.2378/vhn2014.artnd.

Kraus, K. (2015a). Lernort. In J. Dinkelaker \& A. v. Hippel (Hrsg.), Erwachsenenbildung in Grundbegriffen (S. 133-138). Stuttgart: Kohlhammer.

Kraus, K. (2015b). Dem Lernen Raum geben. Planung, Gestaltung und Aneignung pädagogischer Räume. In E. Nuissl \& H. Nuissl (Hrsg.), Bildung im Raum (S. 17-32). Baltmannsweiler: Schneider.

Kreckel, R. (1992). Politische Soziologie der sozialen Ungleichheit. Frankfurt a. M./New York: Campus.

Kronauer, M. (2010). Inklusion und Weiterbildung. Bielefeld: wbv.

Lindmeier, C. (2003). Integrative Erwachsenenbildung. DIE Zeitschrift für Erwachsenenbildung, 4, $28-35$.

Lindmeier, C., \& Lindmeier, B. (2015). Inklusion aus der Perspektive des rechtlichen und ethischen Begründungsdiskurses. Mitteilungen der DGfE, 51, 43-51.

Löw, M. (2001). Raumsoziologie. Frankfurt a.M.: Suhrkamp.

Löw, M., Steets, S., \& Stoetzer, S. (2008). Einführung in die Stadt- und Raumsoziologie (2. Aufl.). Opladen, Farmington Hills: Budrich.

Modes, M.-T. (2016). Raum und Behinderung. Wahrnehmung und Konstruktion aus raumsoziologischer Perspektive. Bielefeld: transcript.

Nuissl v. Rein, E., \& Dollhausen, K. (2011). Kulturen der Programmplanung. Zeitschrift für Pädagogik, 1, 114-129.

Powell, J. W., \& Pfahl, L. (2012). Sonderpädagogische Fördersysteme. In U. Bauer, U. H. Bittlingmeyer \& A. Scherr (Hrsg.), Handbuch Bildungs- und Erziehungssoziologie (S. 721-740). Wiesbaden: VS.

Rau, S. (2013). Räume. Konzepte, Wahrnehmungen, Nutzungen. Frankfurt a.M., New York: Campus.

Reutlinger, C., \& Wigger, A. (2010). Das St. Galler Modell - eine Denkfigur zur Gestaltung des Sozialraums. In C. Reutlinger \& A. Wigger (Hrsg.), Transdisziplinäre Sozialraumarbeit. Grundlegungen und Perspektiven des St. Galler Modells zur Gestaltung des Sozialraums (S. 13-54). Berlin: Frank \& Timme.

Riddell, S., \& Watson, N. (2014). Disability, culture and identity. London, New York: Routledge.

Schäffter, O. (2014). Relationale Zielgruppenbestimmung als Planungsprinzip. Zugangswege zur Erwachsenenbildung im gesellschaftlichen Strukturwandel. Ulm: Klemm \& Oelschläger.

Schäffter, O., \& Ebner von Eschenbach, M. (2015). Inklusion und Exklusion im Diskurs der Erwachsenenbildung. Versuch einer begriffssystematischen Kontextualisierung. Hessische Blätter für Volksbildung, 4, 317-327.

Schrader, J. (2011). Struktur und Wandel der Weiterbildung. Bielefeld: wbv.

Schreiber-Barsch, S. (2015a). Von Sonder-Räumen zu inklusiven Lernorten. Raumordnungen in der Erwachsenenbildung. In C. Bernhard, K. Kraus, S. Schreiber-Barsch \& R. Stang (Hrsg.), Erwachsenenbildung und Raum. Theoretische Perspektiven - professionelles Handeln - Rahmungen des Lernens (S. 193-204). Bielefeld: wbv.

Schreiber-Barsch, S. (2015b). Teilhabe, Inklusion, Partizipation. In J. Dinkelaker \& A. v. Hippel (Hrsg.), Erwachsenenbildung in Grundbegriffen (S. 191-198). Stuttgart: Kohlhammer. 
Schreiber-Barsch, S. (2016). Ist Dabei-sein alles? Inklusive Lernorte der Erwachsenenbildung und die Dialektik von Zugang und Barrieren. In K. Dollhausen \& S. Muders (Hrsg.), Diversität und Lebenslanges Lernen. Aufgaben für die organisierte Weiterbildung (S. 217-229). Bielefeld: wbv.

Schreiber-Barsch, S. (2017). Space is more than place: the urban context as contested terrain of inclusive learning settings for adults and arena of political subjectivation. In H. Sacré \& S. de Visscher (Hrsg.), Learning the city. Cultural approaches to civic learning in urban spaces (S. 67-81). Cham: Springer.

Schreiber-Barsch, S., \& Pfahl, L. (2014). Zur Funktion der „Anderen“: Klassifizierungspraktiken im Bildungswesen und das System des Lebenslangen Lernens. Hessische Blätter für Volksbildung, 3, 232-237.

Schroer, M., \& Wilde, J. (2010). Ort. In C. Reutlinger, C. Fritsche \& E. Lingg (Hrsg.), Raumwissenschaftliche Basics (S. 181-190). Wiesbaden: VS.

Shakespeare, T. (2014). Disability rights and wrongs revisited (2. Aufl.). Abingdon, New York: Routledge.

Snyder, S. L., \& Mitchell, D. T. (2006). Cultural locations of disability. Chicago, London: University of Chicago Press.

Strauss, A.L. (1978). Negotiations. Varieties, contexts, processes, and social order. San Francisco: JosseyBass.

Strauss, A.L., \& Corbin, J. (1996). Grounded Theory: Grundlagen Qualitativer Sozialforschung. Weinheim: Beltz.

Strübing, J. (2014). Grounded theory (3. Aufl.). Wiesbaden: VS.

Theunissen, G., \& Hoffmann, C. (2003). Entwicklung, Theorie und Perspektiven einer Erwachsenenbildung bei Menschen mit Lernschwierigkeiten und mehrfacher Behinderung. In G. Theunissen (Hrsg.), Erwachsenenbildung und Behinderung (S. 45-64). Bad Heilbrunn: Klinkhardt.

UN-BRK (2006). Übereinkommen über die Rechte von Menschen mit Behinderungen. www.behindertenrechtskonvention.info/uebereinkommen-ueber-die-rechte-von-menschen-mitbehinderungen-3101. Zugegriffen: 28. Juli 2017.

United Nations (1948). Resolution der Generalversammlung 217 A (III). Allgemeine Erklärung der Menschenrechte. www.un.org/depts/german/menschenrechte/aemr.pdf. Zugegriffen: 28. Juli 2017.

United Nations (2006). Conventions on the rights of persons with disabilities. https://www.un.org/ development/desa/disabilities/convention-on-the-rights-of-persons-with-disabilities/convention-onthe-rights-of-persons-with-disabilities-2.html. Zugegriffen: 28. Juli 2017.

Zeitschrift für Pädagogik (2016). Thementeil: Raum und Räumlichkeit in der erziehungswissenschaftlichen Forschung. Bd. 1. 